\title{
Artificial Neural Network in Fibres Length Prediction for High Precision Control of Cellulose Refining
}

\author{
Daniele Almonti ${ }^{1}$, Gabriele Baiocco ${ }^{2}$, Vincenzo Tagliaferri ${ }^{1}$ and Nadia Ucciardello ${ }^{1, *}$ \\ 1 Department of Enterprise Engineering "Mario Lucertini", University “Tor Vergata”, Via del Politecnico 1, \\ 00133 Roma, Italy; daniele.almonti@uniroma2.it (D.A.); tagliaferri@mec.uniroma2.it (V.T.) \\ 2 Department of Engineering, University “Roma Tre”, Via Vito Volterra 62, 00146 Roma, Italy; \\ gabriele.baiocco@unioma2.it \\ * Correspondence: nadia.ucciardello@uniroma2.it; Tel.: +39-06-7259-7591
}

Received: 16 October 2019; Accepted: 9 November 2019; Published: 12 November 2019

check for updates

\begin{abstract}
Paper, a web of interconnected cellulose fibres, is widely used as a base substrate. It has been applied in several applications since it features interesting properties, such as renewability, biodegradability, recyclability, affordability and mechanical flexibility. Furthermore, it offers a broad possibility to modify its surface properties toward specifics additives. The fillers retention and the fibres bonding ability are heavily affected by the cellulose refining process that influences chemical and morphological features of the fibres. Several refining theories were developed in order to determine the best refining conditions. However, it is not trivial to control the cellulose refining as different phenomena occur simultaneously. Therefore, it is intuitively managed by experienced papermakers to improve paper structures and properties. An approach based on the machine learning aimed at estimating the effects of refining on the fibres morphology is proposed in this study. In particular, an artificial neural network (ANN) was implemented and trained with experimental data to predict the fibres length as a function of refining process variables. The prediction of this parameter is crucial to obtain a high-performance process in terms of effectiveness and the optimisation of the final product performance as a function of the process parameter. To achieve these results, data mining of the experimental patterns collected was exploited. It led to the achievement of excellent performance and high accuracy in fibres length prediction.
\end{abstract}

Keywords: artificial neural networks; machine learning; cellulose fibres processing; process management; refining optimisation

\section{Introduction}

In its essence, paper is a network of fibres interconnected. Usually, it is filled with several additives aimed at controlling the penetration of coating colours and ink, as well as improving mechanical performance and morphological features. In order to ensure effective additives action and efficient manufacturing processes, a high retention ability of a fibres web is required. Furthermore, for reaching elevated mechanical performance, fibres must feature strong linkages. These paper properties can be deeply affected by the manufacturing process, as it influences the fibres specification, such as morphology and surface chemistry.

The raw materials exploited for the paper production include softwoods, hardwoods and nonwood fibres. The main elements of the fibres may be identified in cellulose, hemicellulose and lignin [1]. Fibres are structured in elementary fibrils, which are twisted around the cell wall axis. The structural formula of microfibrils presents hydroxyl groups in the glucose units, which are responsible for the hydrogen bonding ability and allow for the creation of a strong paper structure [2-4]. Further, since ionisable acidic groups are present in lignin and hemicellulose, when fibres are suspended in 
water, they carry a negative charge. The fibres charge and the electrochemical parameters of a pulp are fundamental in papermaking, as a large number of the interactions between fibres and fillers are charge-induced.

Cellulose refining is a critical step in paper production. During the refining, compression and shear forces are applied to fibres flocs, causing several changes in fibres specifications, such as fibres morphology and distribution of chemicals on the surface of the fibres. Fibres morphological changes have been widely discussed. Particularly, they can be summarised in external and internal fibrillation and fines formation, in addition to fibres shortening and straightening [1]. Internal fibrillation is the effect of delamination of the outer layers of the fibres as a consequence of the cyclic compression action of the forces inside the refining devices [5]. The breakage of the bonds among the three constitutive elements of the fibres causes expansion and swelling of the porous structure inside the cell wall, making the fibres more flexible. In addition, studies demonstrated that layers delaminated during the refining process are major restrains against swelling [6,7]. The leakage of fibrils from the surface of the fibres is defined as external fibrillation, and its main result is the growth of the specific surface area. It is always associated with internal fibrillation and fines formation, since they occur simultaneously. Therefore, it is hard to judge the external fibrillation role within the refining process [8]. Further, growing amounts of charged fines are produced. In [9], fines are defined as the fraction of a pulp that is able to pass through a mesh screen or a perforated plate having a hole diameter of $76 \mu \mathrm{m}$. The secondary fines formation is the effect of external fibrillation or fibres shortening during the refining process. They are different from the primary fines, which come from ray cells and parenchyma cells, and are present also in nonrefined pulps. The primary and secondary fines are usually characterised by a reduced length, generally less than $0.3 \mathrm{~mm}$, and their presence within the pulp causes a greater bonding capacity as well as a reduction in drainage speed [9-11]. The last effect of refining is the fibres length modification as a consequence of the shear and compression forces applied. Fibres lengthening is critical for producing high-quality products, since it results in an enhanced load capacity, stress distribution along the network and elastic modulus [12]. Excessive fibres lengthening or high shear forces may result in fibres shortening. Although the latter is often considered as a detrimental effect, in some rare applications, it may be desirable [13]. As the fibres shortening is related to fines formation, it is difficult to measure accurately the length changes after refining treatment. Usually, length changes are evaluated considering the weighted average lengths before and after refining [14]. In addition, morphological changes of the fibres affect the chemicals and charge distribution on fibres surfaces and walls. Indeed, the morphological changes of fibres make the chemical constituents of the fibres more accessible. However, a relationship between chemical changes and variation of the fibre surface charge during refining has not been established yet [15].

From the above review, it is clear that, in the context of industrial paper production, fibres refining represents one of the greatest challenges in order to obtain high-quality products. Overall, all the aforementioned effects of refining occur simultaneously, influencing each other. It is evident that the refining effects on fibres charge and morphology are extremely complex, since both chemical and mechanical factors are involved. Additionally, they are correlated by means of a nonlinear relationship. Several refining theories were developed in order to determine the most suitable refining control system and the best refining conditions. The most commons are based on the energy applied to the fibres during the process. Therefore, it is the most exploited variable to define the refining intensity [16-18]. These theories also consider parameters such fibre lengths, coarseness, refining disks geometry and rotation speeds, in addition to pulp consistency. However, other theories consider the forces in order to characterise the refining action [19-21].

Although these several refining theories and methods to explore how a three-dimensional paper structure is related to macroscopic paper properties are now emerging, alternative indirect methods were applied over years. However, no theories concerning the morphological modification of refined fibres were reported. Therefore, the manufacturing process is often managed by experienced papermakers that know intuitively how to vary the refining settings to improve the paper properties. 
The knowledge is important, despite being more empirical than scientific. In the context of artificial intelligence (AI), expert systems and artificial neural networks (ANNs) have been widely employed to exploit the empirical knowledge in the management of industrial production systems. The ANNs have been widely applied over years; particularly, they were applied in modelling, pattern classification and clustering tasks [22]. There are several application fields, including aid of decision-making processes [23-26], classification tasks [27-29], phenomena and processes prediction [30-34], design optimisation $[35,36]$ and materials characterisation $[37,38]$.

Several works in the literature investigate an ANN approach to paper-making. These methods mainly concern the application of neural models for paper properties prediction [39-43]; others deal with pulping process control $[44,45]$. To the best of our knowledge, no work concerning the prediction of fibres morphology, and specifically fibres lengths, by means of AI has been presented.

The objective of the proposed work is the implementation of a machine learning system addressed towards the control of the refining process. Particularly, the implementation of an ANN aimed at predicting the length of the outgoing fibres from a refining device is presented. The possibility of foreseeing one of the fundamental morphological characteristics of the fibres would be crucial for optimising the product performance and manufacturing the process efficiency. In this respect, the collection and the analysis of an experimental dataset allowed for the training of the ANN implemented. The result achieved showed an excellent ability in the prediction of the fibres length after a refining process on the basis of the main process parameters involved in the paper production. Therefore, the application of ANNs is a valuable tool for the innovation of the traditional control methods aimed at optimising the manufacturing process and the final products performance.

\section{Materials and Methods}

The refining analysis was carried out considering a system composed of four conical refiners, as represented in Figure 1.

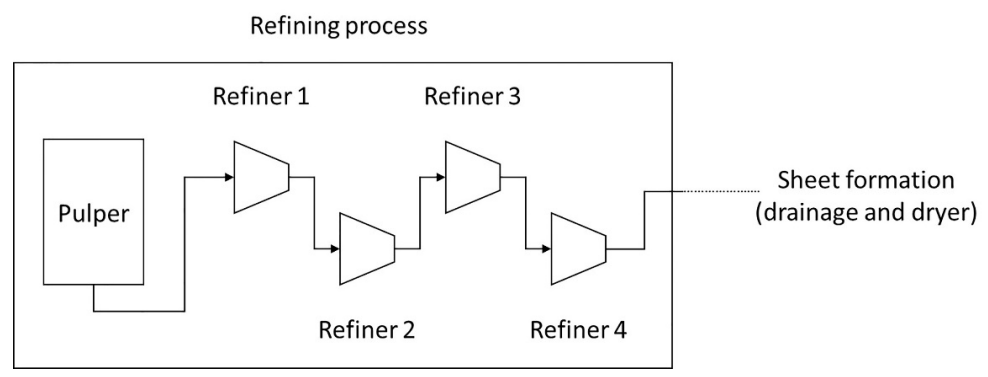

Figure 1. Schematic of a refining process.

Outgoing pulp samples from refiners and outgoing untreated pulp from a pulper were collected and analysed. Initially, the main variables affecting the refining process were identified. In particular, three typologies of variables were defined. The first group of variables concerned the pulp composition in terms of fibres content and fillers amount, which remained constant for all the refining phases. The fibres were classified both qualitatively and quantitatively, reporting their amounts as a percentage of the total quantity of the pulp. The fibres qualities exploited in the paper production during analysis performed were as follows: Pacifico, FSC Cenibra, A3F from Aracruz, FSC Celbi, Arauco Radiata, FSC BTMP Waggeryd and FSC CMPC Guaiba. Instead, the fillers amount was considered as the total amount of the different additives exploited to mix with the pulp. This simplification was made with the aim of reducing the variables number. Furthermore, the titanium dioxide was a preponderant element among the additives exploited in the different pulps considered. The additives considered for the process analysis were iron oxides, kaolin and inks. Other additives exploited but not considered for the analysis, as constant during the experimental investigation, were resins and lye. 
After that, the refining process parameters were considered. The net refining power, which was the difference between load power and no-load power consumed by refiners, and the pulp flow were chosen, as their combination was an index of the refining intensity. Furthermore, the refining bar geometry of the conical refining disks, which was the shape of the bars providing for pulp refining and transport of fibre suspension, and their wear rate considered as the hours of operation were taken into account. In particular, the refiners exploited in this work were as follows: Parason SF (Parason, Aurangabad, India), Parason PA-2815SF (Parason, Aurangabad, India), Airaghi SF-2063019 (Officine Airaghi (S.R.L.), San Giovanni Lupatoto, Italy), Airaghi XSF-2063019 (Officine Airaghi (S.R.L.), San Giovanni Lupatoto, Italy) and Airaghi SF-20633019 (Officine Airaghi (S.R.L.), San Giovanni Lupatoto, Italy). In order to create a dataset suitable for the neural network training, five refining disk geometries exploited for the production were considered qualitatively. The last input parameter concerned the fibres morphology. Specifically, it is the mean length of the incoming fibres. The length of the fibres was chosen also as a parameter for the process control, since length modification is one of the main effects of the refining and a valuable index to evaluate the process effectiveness. The length measures were carried out by means of a fibres tester, Lorentzen \& Wettre 912 plus (AB Lorentzen \& Wettre, Kista, Sweden). It provides an automated fibres quality measurement of a pulp for fast classification and detection of fibres features, such as mean lengths, widths, areas, perimeters and shape factors, in primary and secondary fines amounts. In particular, the device calculates the weighted average fibre length, evaluated as the sum of individual fibre lengths squared divided by the sum of the individual fibre lengths. The boundary values of the variables considered in the dataset are reported in Table 1.

Table 1. Values of the variables analysed.

\begin{tabular}{cc}
\hline Variables & Min/Max \\
\hline Fibres composition (1-7) & $0 / 1$ \\
Fillers amount & $850 / 1538[\mathrm{~kg}]$ \\
Net power & $0 / 158[\mathrm{kw}]$ \\
Pulp flow rate & $760 / 1538[\mathrm{~L} / \mathrm{min}]$ \\
Wear rate & $1 / 2352[\mathrm{~h}]$ \\
Fibres length & $0.768 / 1.140[\mathrm{~mm}]$ \\
\hline
\end{tabular}

With the aim of acquiring examples to be exploited in the neural network training, a subdataset was acquired for each refiner involved in the cellulose refining, as represented in Figure 2.

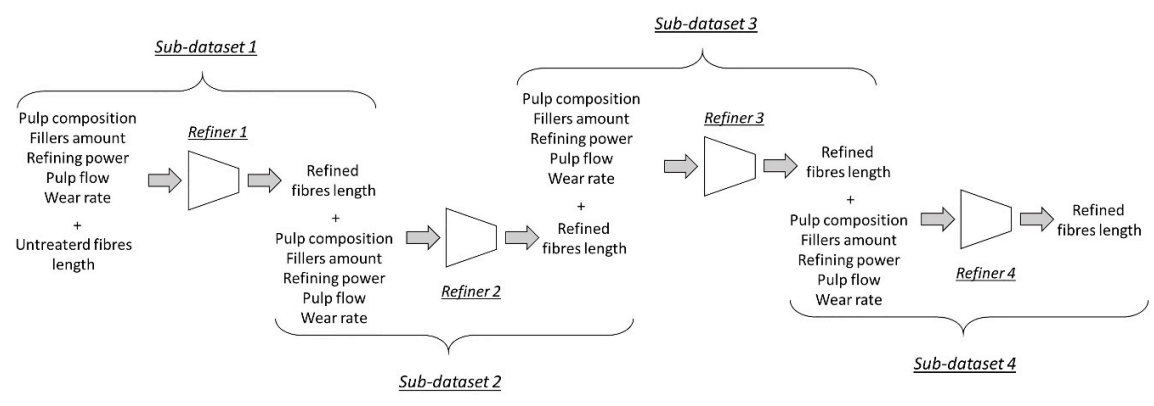

Figure 2. Subdataset creation scheme.

Under the simplifying hypothesis that the refiners process the fibres in the same way, the experimental data extrapolated from each refiner were exploited to build a single dataset to be used in the ANN training. Additionally, the refiners were considered independent, so that, for a given stage, the effect of the previous refining step could be neglected. This is not rigorous, as there is a limit in the fibres lengthening during the refining and therefore in the energy that can be globally supplied to the fibres. The energy transferred to the fibres during the single refining step is low and does not affect the 
following refining stage. This allows for the development of a single neural network for the prediction of length modification after each step of the refining line considered in this study.

The first ANN developed, named ANN1, is represented in Figure 3.

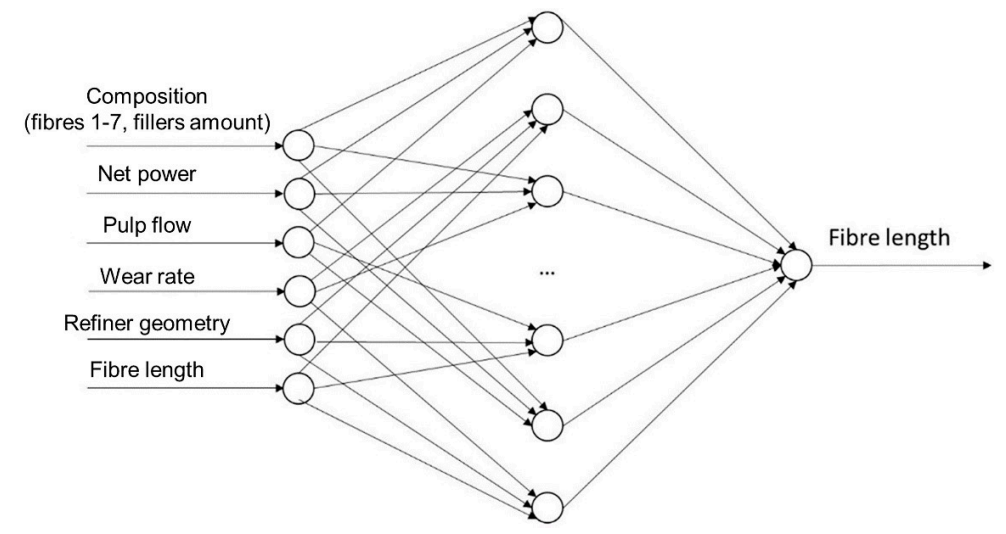

Figure 3. Schematic of the first artificial neural network (ANN1) structure.

It was trained by means of the Broyden-Fletcher-Goldfarb-Shanno (BFGS) backpropagation algorithm exploiting 72 patters collected during the experimental phases previously described. The algorithm was chosen, because a quasi-Newtown method is one of the most suitable techniques for function fitting. Like any of Newton-like methods, BFGS uses the quadratic Taylor approximation of the objective function about a generic point $x_{n}$, which can be written as Equation (1):

$$
f\left(x_{n}+\delta x\right)=f\left(x_{n}\right)+g_{n}^{T} \delta x+\frac{1}{2} \delta x^{T} H_{n} \delta x
$$

where

$$
\begin{aligned}
& g_{n}=\nabla f\left(x_{n}\right) \\
& x_{n}=\nabla^{2} f\left(x_{n}\right)
\end{aligned}
$$

The necessary condition for a minimum was described as:

$$
\begin{gathered}
\nabla f\left(x_{n}\right)=g_{n}+H_{n} \delta x=0 \\
\delta x=-H_{n}^{-1} g_{n}
\end{gathered}
$$

In an iterative process, the variables update was expressed as:

$$
x_{n+1}=x_{n}-H_{n}^{-1} g_{n}
$$

In quasi-Newton methods, the idea is to use matrices that approximate, instead of computing, the Hessian matrix Hn for a reduced computational effort. Particularly, the BFGS method estimates the Hessian matrix with the following Equation (7):

$$
H_{n+1}=H_{n}-\frac{\left(H_{n} s_{n}\right)\left(s_{n} H_{n}\right)^{T}}{H_{n} s_{n} s_{n}^{T}}+\frac{q_{n} q_{n}^{T}}{q_{n}^{T} s_{n}}
$$

A connection between an input layer and a hidden layer was the tangent function, as it is most suitable in a hidden layer for ANNs aimed at function fitting. Although the pure linear transfer function is the most suitable for the connection of the hidden and output layers in order not to shorten a wide output range in a short interval, a logarithmic transfer function was applied. This was possible as the output values characterising the patterns in the dataset were compressed in a very short range. The numbers of neurons of the input and output layers were 13 and 1, respectively, representing the 
variables analysed. The hidden layer counted 25 neurons, which was the size giving the best prediction performance. It was the results of the test of several neural networks featuring the same architecture and different hidden sizes. The training of the network was performed exploiting $70 \%$ of the dataset for the training and the remaining $30 \%$ for the network validation. The stop criterion imposed was the maximum number of epochs equal to 2000 or the minimum square error equal than $1 \times 10^{-10}$.

In order to enhance the neural network training, the dataset was treated by means of statistical tools. In fact, the process data exploited in the ANN1 training were handwritten in a specific paper register, as the IT (Information Technology) tools for data collection were missing in the paper mill object of the case study. This is a major source of uncertainty, which may cause a drop in the ANN performance. Particularly, a principal component analysis (PCA) was performed, excluding the data concerning the pulp fibres composition and the refiner geometry. It was mostly aimed at reducing the variance within the data introduced by inaccurate records of the raw materials quantities and the process parameters settings. Furthermore, the consequent input variables reduction aids the evaluation of the error surface, enhancing the ANN performance, reducing the ANN architecture's size and the weights number. Additionally, the reduction of the variables number was approached, evaluating the correlation coefficients and performing a scatterplot analysis. At a later stage, a single-linkage hierarchical clustering (HC) was exploited, evaluating the Euclidean distances among the data for the outlier detection.

The resulting modified dataset was applied for the training of the second artificial neural network (ANN2), of which the structure is represented in Figure 4.

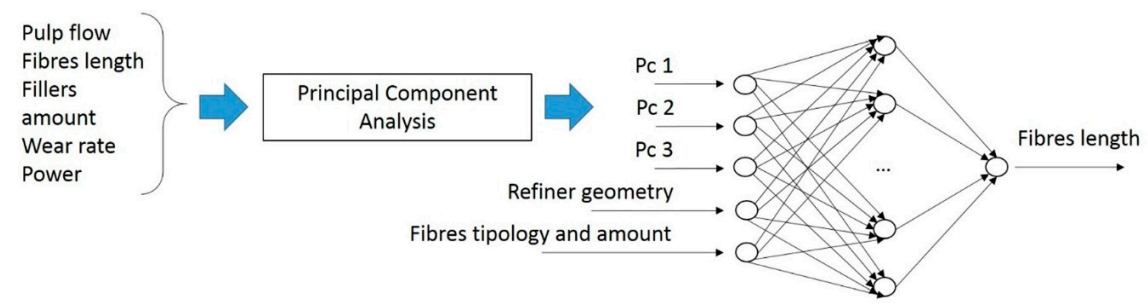

Figure 4. Schematic of the second artificial neural network (ANN2) structure.

It had an input layer featuring a reduced number of neurons with respect to the ANN1 implemented. In particular, the fibres typology and refiner geometry variables were kept unchanged. Instead, the pulp flow, fibres length, fillers amount, wear rate and refining power were elaborated by means of a PCA, which led to the definitions of three principal components. Therefore, the input layer counted 11 neurons, which referred to the input variable, while the output layer presented a single neuron in the representation of the fibres length. The hidden layer counted 7 neurons, which is the number of neurons to achieve the best performance. Again, the transfer functions between the layers were the tangent sigmoid and the logarithmic sigmoid transfer function for the output and hidden layers, respectively. As the size of this this ANN architecture was decreased with respect to the first attempt, the training algorithm was modified. In particular, the algorithm exploited was the the Levenberg-Marquardt algorithm, since it gives the best performances with small-size architecture when accurate training is required. The weight estimation was performed with the following equation, which produced a fester convergence of the training:

$$
x_{n+1}=x_{n}-(H-\lambda \operatorname{diag}[H])^{-1} g_{n}
$$

For this reason, the minimum mean squared error and the epoch number were reduced with respect to the first ANN and set to $10^{-10}$ and 2000, respectively. The training was performed with $70 \%$ of the data left from the dataset cleaning, which was 52 patterns, while the test was conducted with the remaining $30 \%$ of the data. The split of the dataset was random. 
While the performance of the networks was evaluated by means of the mean squared error, the error committed was evaluated as a ratio of the difference between the actual and predicted fibres lengths with an actual length as reported in the following Equation (9):

$$
E \%=\frac{L_{p}-L_{a}}{L_{a}} \times 100
$$

where $L_{p}$ is the predicted length and $L_{a}$ is the actual length.

\section{Results and Discussion}

The results achieved with the ANN1 are reported in Figure 5, where E\% committed for each pattern included in the validation set is reported.

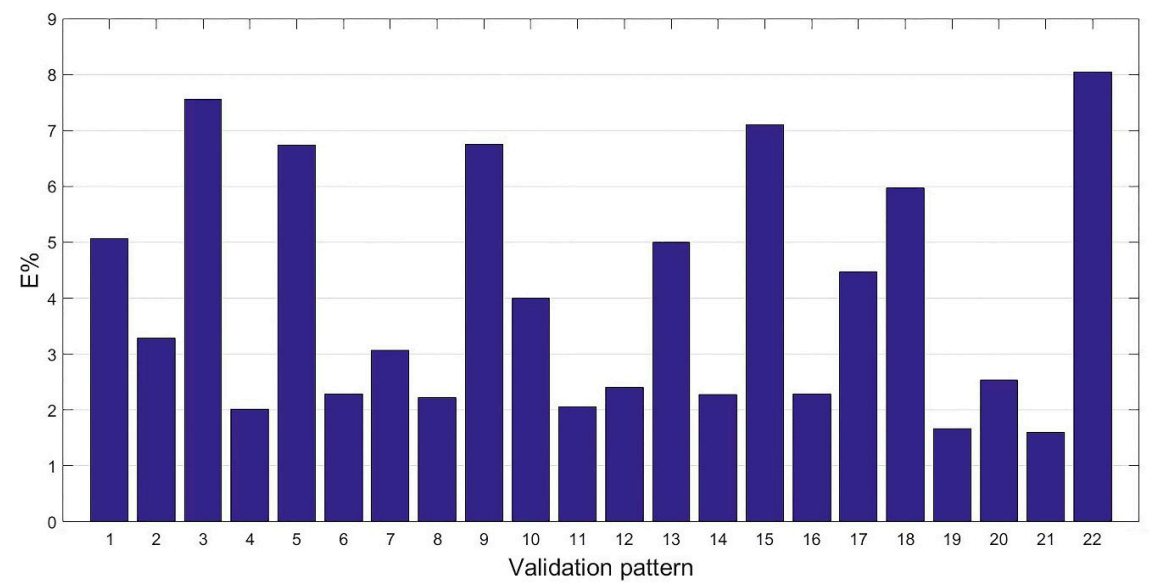

Figure 5. Validation results of the ANN1.

The mean percentage error committed by the network was $4.01 \%$, which resulted in a difference between the actual and predicted lengths of about a hundredth of a millimetre the error committed by the network was not constant on the entire validation set. The validation featured a standard deviation of 2.16 with examples featuring errors up to $8 \%$. Additionally, the regression plot of the model was performed and reported in Figure 6, which confirms the indication obtained by the E $\%$ analysis. Indeed, a rather low value of $R^{2}=0.67109$ indicates the model does not represent a generalisation of the refining process under analysis.

These results may be caused by the presence of inaccurate patterns within the dataset due to the lack of information technologies. Indeed, the inaccurate data within the training set did not allow for the evaluation of a precise model. Therefore, the ANN created cannot replicate the actual manufacturing process and then offers an insufficient generalisation ability with a consequent high standard deviation of the mean percentage error. Furthermore, the outliers in the validation set may be responsible for those patterns featuring the highest error. Indeed, being outliers, those patterns cannot fit the model built in the training phase and, when the network is interrogated with the unknown data, produce elevated errors.

As mentioned, both the problems were addressed with statistical analysis methods, HC and PCA. The statistical analysis comprised the evaluation of the correlation coefficient, reported in Table 2, and a scatterplot analysis, reported in Figure 7 . The correlation coefficient showed that no linear correlation characterised the dataset. The same occurred with the scatterplot analysis, as no trend was highlighted, and the data were plotted as a cloud of points. Therefore, a nonlinear correlation was detected. 


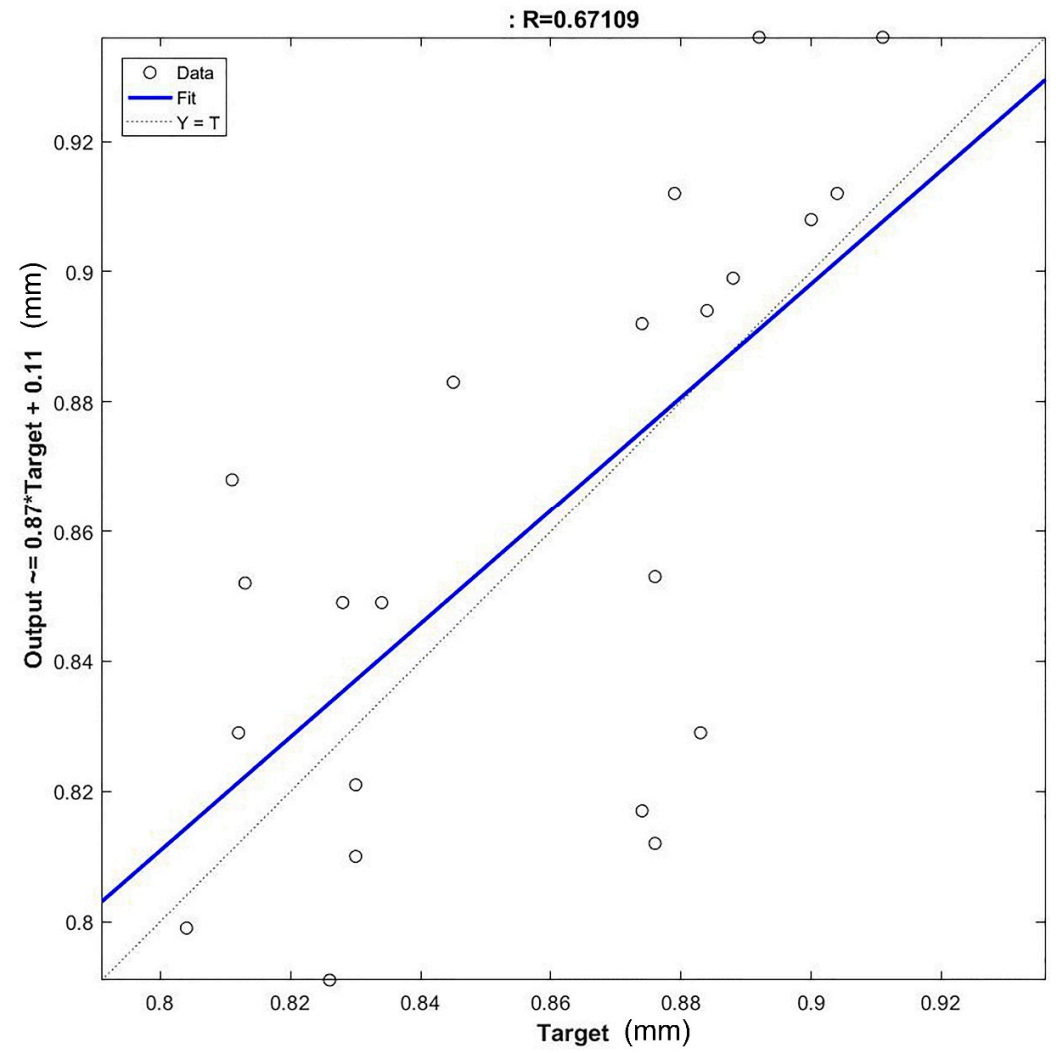

Figure 6. Regression plot of the ANN1 trained with the untreated data.

Table 2. Correlation coefficients.

\begin{tabular}{cccccc}
\hline & Pulp Flow & Fillers & Power & Time & Length \\
\hline Pulp flow rate & 1 & 0.061 & 0.272 & 0.081 & -0.300 \\
Fillers amount & 0.061 & 1 & 0.029 & 0.080 & -0.334 \\
Power & 0.272 & 0.029 & 1 & -0.261 & -0.010 \\
Operation hours & 0.081 & 0.080 & -0.261 & 1 & -0.148 \\
Length & -0.300 & -0.334 & -0.010 & -0.148 & 1 \\
\hline
\end{tabular}

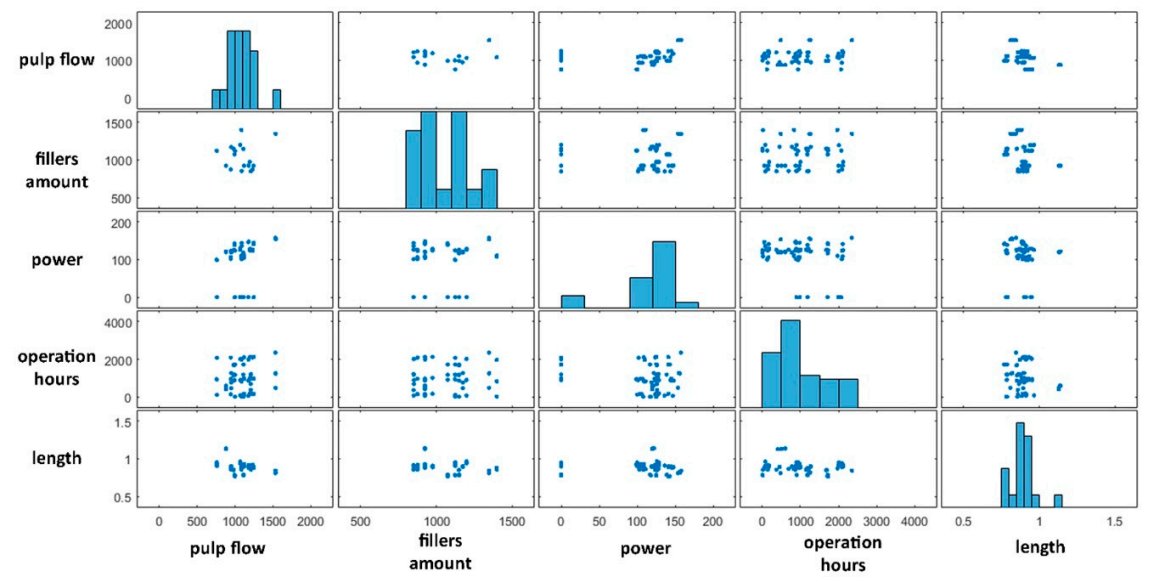

Figure 7. Scatter plot results.

Therefore, the dataset analysis proceeded with a PCA, of which the results are reported in Figures 8 and 9. 


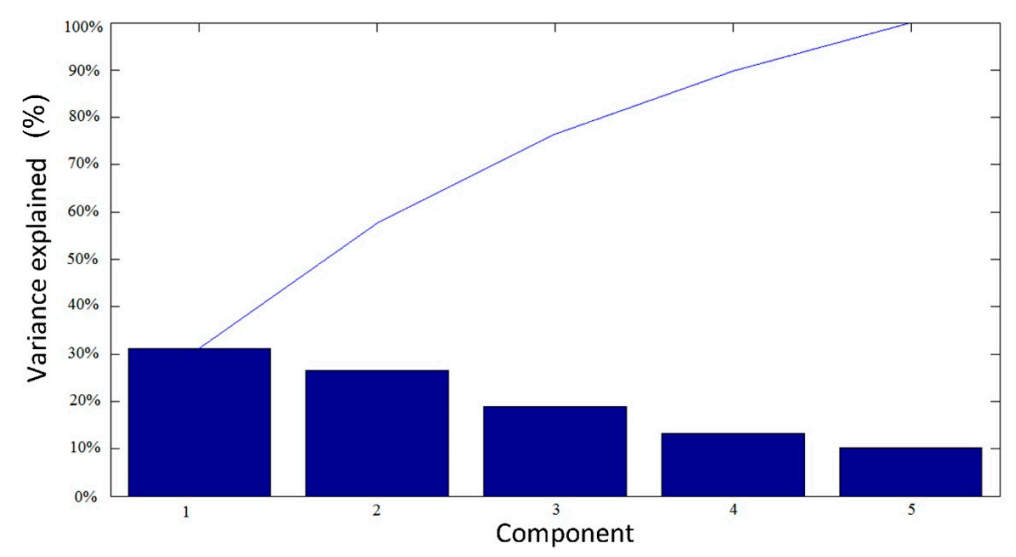

Figure 8. Principal component plotted as a function of the explained variance.

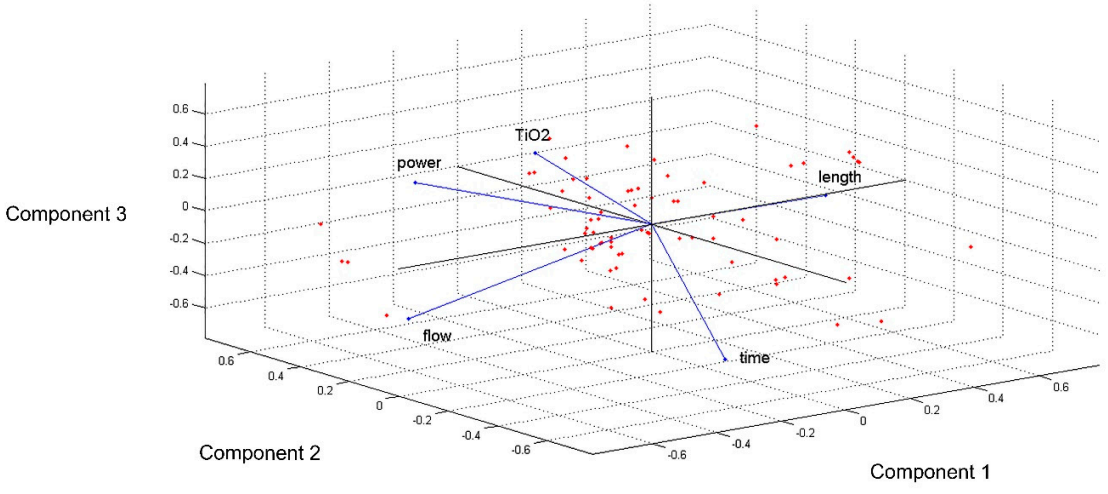

Figure 9. Patterns distribution in the principal components space.

Figure 8 highlights the results of the analysis, which was the variance explained by the principal components. Particularly, $82 \%$ the principal components can be compressed with the exploitation of three principal components; therefore, the variables included in the study were substituted by the first, second and third principal components.

At a later stage, the distribution of the patterns among the principal component space was analysed.

As reported in Figure 9, despite a central core of examples, aseveral patterns spreading to the boundary of the cloud of data analysed can be individuated. These patterns could be the outliers, and with the aim of detecting them, an HC was performed. The result is reported in Figure 10.

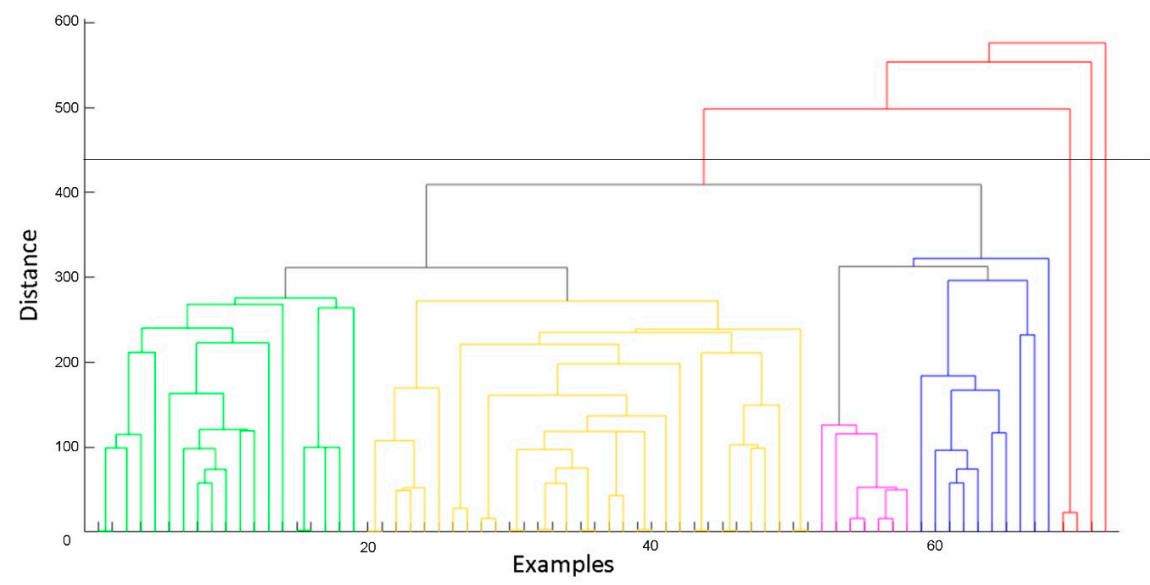

Figure 10. Hierarchical clustering results. 
In the HC clustering reported while the horizontal axis represents the patterns or groups of patterns within the dataset, the vertical axis represents their geometrical distances in the multidimensional space of the variables. The shorter are the vertical segments connecting the patterns, and the closer and more similar are the examples analysed. It is evident how the dataset could be divided into four similar groups, where the distances among the examples are comparable. They can be associated to four similar kinds of paper produced in the paper mill during the study performed. Four subgroups of examples were characterised by an elevated distance from the other groups. These subgroups, which counted a total number of 19 patterns, were considered as outliers and not included in the dataset for the network training.

With the new dataset comprising the principal components instead of the actual variables and without the outliers detected, the training of ANN2 was performed. The validation results obtained are reported in Figure 11.

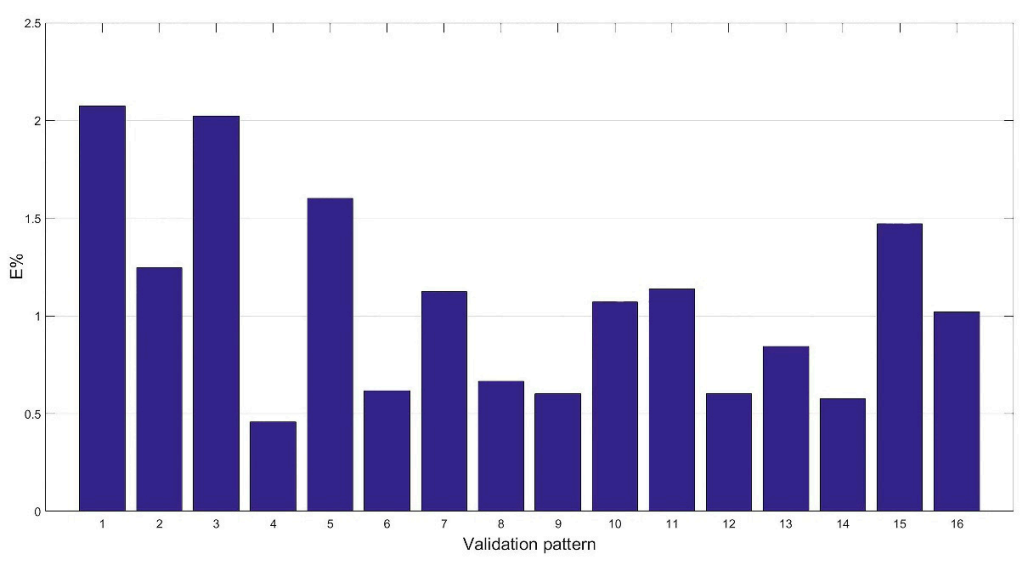

Figure 11. Validation results of the ANN2.

It is evident the error committed was reduced. The network featured a mean error of $1.07 \%$ with a standard deviation of 0.5 . Differently from the previous network implemented, the error was constant among the entire validation set, with a peak error comparable with the mean error of the previous network. It is inferable that the problems related to the dataset and the complexity of the system under examination were correctly addressed with the statistical analysis. The reduction of the input variables led to the definition of a simplified error surface, which allowed for the creation of a precise model capable to give a low mean percentage error with a reduced standard deviation. This is also visible considering the reduced number of neurons in the hidden layer necessary for the creation of an accurate model of the refining system. Additionally, the elimination of the outliers allowed obtaining a homogeneous dataset that led to the elimination of the highest error in the validation set. To validate the results obtained with the ANN2, a regression plot was performed to assess the similarity of the neural model built with the experimental data. It is reported in Figure 12.

An $\mathrm{R}^{2}$ value of 0.98 indicates the goodness of the model built, and therefore the model produced by ANN2 can be considered highly reliable. This is confirmed by the ANOVA, of which the results are reported in Table 3, which state the significance of the ANN model produced.

Table 3. ANOVA for the neural network model.

\begin{tabular}{cccccc}
\hline Source & DF & SS & MS & F-value & $P$-value \\
\hline Model & 1 & 0.021407 & 0.021407 & 387.57 & $1.3317 \times 10^{-11}$ \\
Residual & 14 & 0.00077327 & $5.5234 \times 10^{-5}$ & & \\
Total & 15 & 0.02218 & 0.0014787 & & \\
\hline
\end{tabular}


Later, the results of the network were analysed, considering not only the prediction accuracy but also the overall effect of the refining on the fibres. Particularly, the interest was to state the network ability in detecting cutting or stretching phenomena. For this reason, the difference between the initial and final lengths of the fibres was computed with the actual and predicted values to verify if they featured the same sign. In Table 4, the validation examples with a nonconcordant length difference are reported.

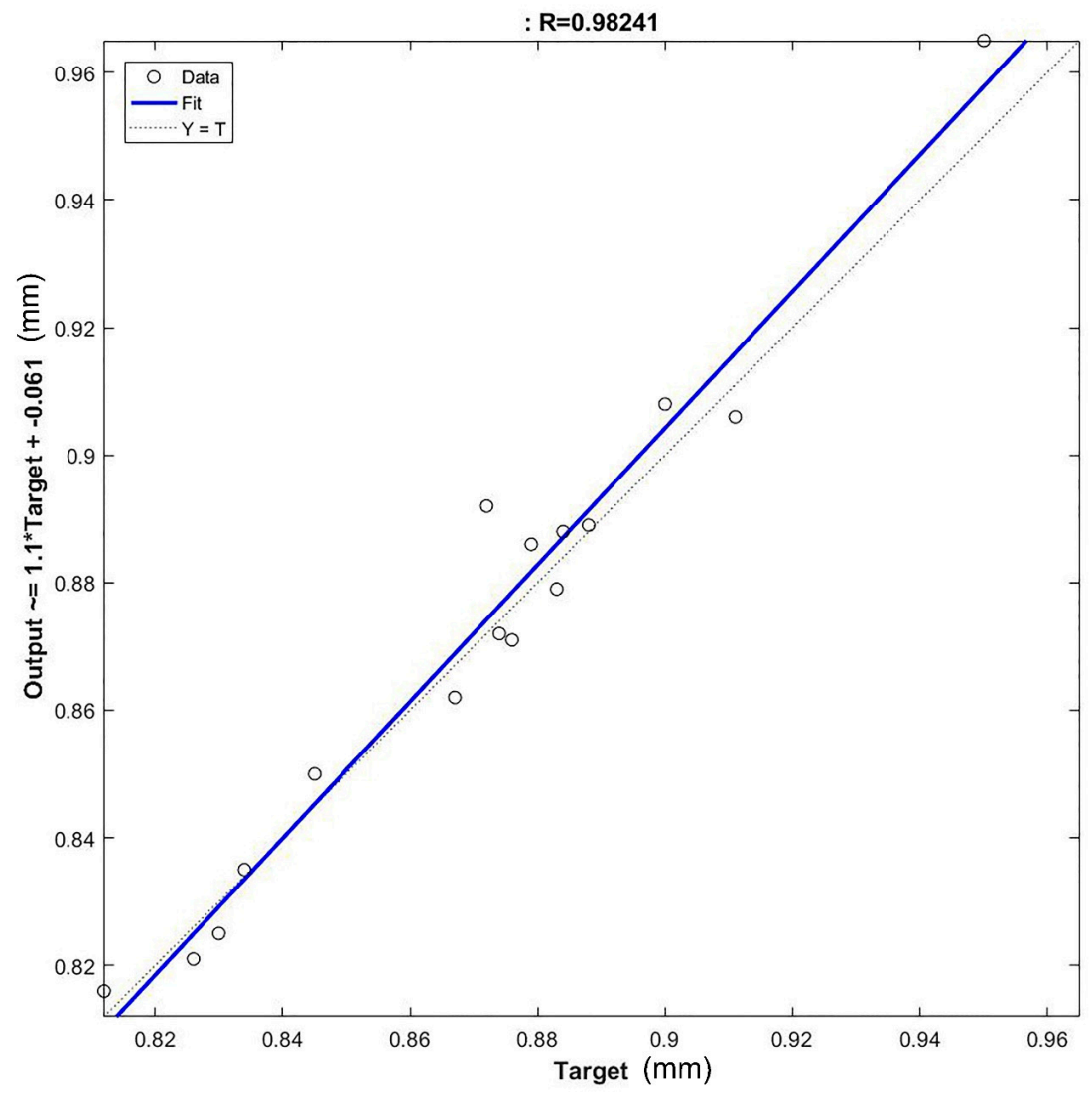

Figure 12. Regression plot of the ANN2 trained with the treated data.

Table 4. Validation examples with a nonconcordant length difference.

\begin{tabular}{ccccc}
\hline $\begin{array}{c}\text { Input } \\
\mathbf{( m m})\end{array}$ & $\begin{array}{c}\text { Target } \\
\mathbf{( m m})\end{array}$ & $\begin{array}{c}\text { Prediction } \\
\mathbf{( m m})\end{array}$ & $\begin{array}{c}\text { Actual Delta } \\
\mathbf{( m m})\end{array}$ & $\begin{array}{c}\text { Predicted Delta } \\
\mathbf{( m m})\end{array}$ \\
\hline 0.883 & 0.872 & 0.892 & -0.009 & +0.009 \\
0.951 & 0.950 & 0.965 & -0.001 & +0.014 \\
0.867 & 0.867 & 0.862 & 0 & -0.005 \\
\hline
\end{tabular}

The example with a difference between the actual and predicted lengths concerned the process where the fibres were untreated. Indeed, all these examples feature a delta in the order of a thousandth of a millimetre. The accuracy of the network is in the order of a hundredth of a millimetre, and the variation of the length in a smaller range was not evaluated correctly. However, it is inferable that the networks have a great capacity in predicting the overall effect of refining on fibres.

\section{Conclusions}

In the present paper, a case study with the development of a machine learning system for optimising a cellulose-refining system was proposed. An ANN was implemented and trained to model the fibres refining, a critical stage of paper manufacturing affecting both the paper mill effectiveness and the final product performance. On the basis of the process parameters exploited in the production 
process management, the ANN proposed allows for the prediction of the fibres length caused by the mechanical refining. The experimental dataset was treated by means of statistical tools; this led to the achievement of outstanding results in fibres length prediction. As this parameter is critical to achieve specific paper properties, its prediction allows for the optimisation of the additives amount and the process parameter to obtain specific properties of the final product.

Author Contributions: All the authors contributed equally to the various aspect of this work.

Funding: This research received no external funding.

Conflicts of Interest: The authors declare no conflicts of interest.

\section{References}

1. Gharehkhani, S.; Sadeghinezhad, E.; Kazi, S.N.; Yarmand, H.; Badarudin, A.; Safaei, M.R.; Zubir, M.N.M. Basic effects of pulp refining on fiber properties-A review. Carbohydr. Polym. 2015, 115, 785-803. [CrossRef] [PubMed]

2. Abe, K.; Yano, H. Comparison of the characteristics of cellulose microfibril aggregates of wood, rice straw and potato tuber. Cellulose 2009, 16, 1017-1023. [CrossRef]

3. Chinga-Carrasco, G. Cellulose fibres, nanofibrils and microfibrils: The morphological sequence of MFC components from a plant physiology and fibre technology point of view. Nanoscale Res. Lett. 2011, 6, 417. [CrossRef] [PubMed]

4. Samyn, P.; Barhoum, A.; Öhlund, T.; Dufresne, A. Review: Nanoparticles and nanostructured materials in papermaking. J. Mater. Sci. 2018, 53, 146-184. [CrossRef]

5. El-Sharkawy, K.; Haavisto, S.; Koskenhely, K.; Paulapuro, H. Effect of fiber flocculation and filling design on refiner loadability and refining characteristics. BioResources 2008, 3, 403-424. [CrossRef]

6. Maloney, T.; Paulapuro, H. The formation of pores in the cell wall. J. Pulp Pap. Sci. 1999, 25, 430.

7. Rusu, M.; Mörseburg, K.; Gregersen, Ø.; Yamakawa, A.; Liukkonen, S. Relation between fibre flexibility and crosssectional properties. BioResources 2011, 6, 641-655. [CrossRef]

8. Clark, J. Fibrillation, free water and fiber bonding. Tappi J. 1969, 52, 335-340.

9. Ferreira, P.J.; Matos, S.; Figueiredo, M.M. Size Characterization of Fibres and Fines in Hardwood Kraft Pulps. Part. Part. Syst. Charact. 1999, 16, 20-24. [CrossRef]

10. Wistara, N.; Young, R.A. Properties and treatments of pulps from recycled paper. Part I. Physical and chemical properties of pulps. Cellulose 1999, 6, 291-324. [CrossRef]

11. Hubbe, M.A.; Heitmann, J.A. Review of factors affecting the release of water from cellulosic fibers during paper manufacture. BioResources 2007, 2, 500-533. [CrossRef]

12. Hartler, N. Aspects on curled and microcompressed fibers. Nord. Pulp Pap. Res. J. 1995, 10, 4-7. [CrossRef]

13. Stoere, P.; Nazhad, M.; Kerekes, R. An experimental study of the effect of refining on paper formation. Tappi J. 2001, 84, 1-9.

14. Batchelor, W.J.; Kure, K.-A.; Ouellet, D. Refining and the development of fibre properties. Nord. Pulp Pap. Res. J. 1999, 14, 285-291. [CrossRef]

15. Fardim, P.; Durán, N. Modification of fibre surfaces during pulping and refining as analysed by SEM, XPS and ToF-SIMS. Colloids Surf. A Physicochem. Eng. Asp. 2003, 223, 263-276. [CrossRef]

16. Lumiainen, J. New theory can improve practice. Pulp Pap. Int. 1990, 32, 46-47.

17. Olejnik, K. Impact of Pulp Consistency on Refining Process Conducted under Constant Intensity Determined by SEL and SEC Factors. BioResources 2013, 8. [CrossRef]

18. Kerekes, R.J. Characterization of pulp refiners by a C-factor. Nord. Pulp Pap. Res. J. 1990, 5, 3-8. [CrossRef]

19. Kerekes, R.J. Energy and forces in refining. J. Pulp Pap. Sci. 2010, 36, 10-15.

20. Kerekes, R.J. Mechanical Pulping: Force-based characterization of refining intensity. Nord. Pulp Pap. Res. J. 2011, 26, 14-20. [CrossRef]

21. Kerekes, R.J.; Senger, J. Characterizing refining action in low consistency refiners by forces on fibres. J. Pulp Pap. Sci. 2006, 32, 1-8.

22. Basheer, I.; Hajmeer, M. Artificial neural networks: Fundamentals, computing, design, and application. J. Microbiol. Methods 2000, 43, 3-31. [CrossRef] 
23. Shafeek, H.; Gadelmawla, E.; Abdel-Shafy, A.; Elewa, I. Automatic inspection of gas pipeline welding defects using an expert vision system. NDT E Int. 2004, 37, 301-307. [CrossRef]

24. Walczak, S.; Velanovich, V. Improving prognosis and reducing decision regret for pancreatic cancer treatment using artificial neural networks. Decis. Support Syst. 2018, 106, 110-118. [CrossRef]

25. Anagnostou, T.; Remzi, M.; Djavan, B. Artificial neural networks for decision-making in urologic oncology. Rev. Urol. 2003, 5, 15-21. [CrossRef]

26. Yu, X.; Ye, C.; Xiang, L. Application of artificial neural network in the diagnostic system of osteoporosis. Neurocomputing 2016, 214, 376-381. [CrossRef]

27. Kılıç, K.; Boyacı, İ.H.; Köksel, H.; Küsmenoğlu, İ. A classification system for beans using computer vision system and artificial neural networks. J. Food Eng. 2007, 78, 897-904. [CrossRef]

28. Bakhshipour, A.; Jafari, A. Evaluation of support vector machine and artificial neural networks in weed detection using shape features. Comput. Electron. Agric. 2018, 145, 153-160. [CrossRef]

29. Lorenz, C.; Ferraudo, A.S.; Suesdek, L. Artificial Neural Network applied as a methodology of mosquito species identification. Acta Trop. 2015, 152, 165-169. [CrossRef] [PubMed]

30. Baiocco, G.; Tagliaferri, V.; Ucciardello, N. Neural Networks Implementation for Analysis and Control of Heat Exchange Process in a Metal Foam Prototypal Device. In Procedia CIRP; Elsevier: Amsterdam, The Netherlands, 2017; Volume 62, pp. 518-522.

31. Bre, F.; Gimenez, J.M.; Fachinotti, V.D. Prediction of wind pressure coefficients on building surfaces using artificial neural networks. Energy Build. 2018, 158, 1429-1441. [CrossRef]

32. Lucignano, C.; Montanari, R.; Tagliaferri, V.; Ucciardello, N. Artificial neural networks to optimize the extrusion of an aluminium alloy. J. Intell. Manuf. 2010, 21, 569-574. [CrossRef]

33. Almonti, D.; Ucciardello, N. Design and Thermal Comparison of Random Structures Realized by Indirect Additive Manufacturing. Materials 2019, 12, 2261. [CrossRef] [PubMed]

34. Missori, S.; Sili, A.; Ucciardello, N. Process parameters optimization of laser beam welded joints by neural network. Mater. Manuf. Process. 2008, 23, 169-174. [CrossRef]

35. Silva, N.; Ferreira, L.M.D.F.; Silva, C.; Magalhães, V.; Neto, P. Improving Supply Chain Visibility with Artificial Neural Networks. Procedia Manuf. 2017, 11, 2083-2090. [CrossRef]

36. Kim, S.H.; Vu, T.M.; Pyeon, C.H. A preliminary study on applicability of artificial neural network for optimized reflector designs. Energy Procedia 2017, 131, 77-85. [CrossRef]

37. Costanza, G.; Tata, M.E.; Ucciardello, N. Superplasticity in PbSn60: Experimental and neural network implementation. Comput. Mater. Sci. 2006, 37, 226-233. [CrossRef]

38. Baiocco, G.; Ucciardello, N. Neural network implementation for the prediction of secondary phase precipitation and mechanical feature in a duplex stainless steel. Appl. Phys. A 2019, 125, 20. [CrossRef]

39. Gyaneshwar, M.; Hart, D.; Scott, W.E. Development of Mathematical Models for Predicting Sizing, Strength, and Opacity on the Miami University Pilot Paper Machine. In 2000 TAPPI Papermakers Conference; TAPPI Press: Atlanta, GA, USA, 2000.

40. Olejnik, K.; Ciesielski, K. Neural network model of pulp refining process. Chem. Process Eng. 2004, 25, 1411-1416.

41. Scharcanski, J.; Dodson, C.T.J. Neural network model for paper forming process. In Proceedings of the Pulp and Paper Industry Technical Conference, Birmingham, AL, USA, 10-14 June 1996; pp. 236-255.

42. Nieminen, P.; Kärkkäinen, T.; Luostarinen, K.; Muhonen, J. Neural Prediction of Product Quality Based on Pilot Paper Machine Process Measurements. In ICANNGA'11-10th International Conference on Adaptive and Natural Computing Algorithms; Springer: Berlin/Heidelberg, Germany, 2011; pp. 240-249.

43. Ciesielski, K.; Olejnik, K. Application of Neural Networks for Estimation of Paper Properties Based on Refined Pulp Properties. Fibres Text. East. Eur. 2014, 22, 126-132.

44. Zhu, J.; Xia, Q.; Rao, M.; Zurcher, J. A Neural Network for Modeling Pulp Process. Pulp Pap. Can. 1997, 98, 35-38.

45. Dayal, B.S.; MacGregor, J.; Taylor, P.A. Application of Feedforward Neural networks and Partial Least Squares Regression to Modeling Kappa Number in a Continuous Kamyr Digester. Pulp Pap. Can. 1994, 95, $26-32$.

(C) 2019 by the authors. Licensee MDPI, Basel, Switzerland. This article is an open access article distributed under the terms and conditions of the Creative Commons Attribution (CC BY) license (http://creativecommons.org/licenses/by/4.0/). 